\title{
Perfiles de participantes en MOOC sobre Aula Invertida \\ Profiles of participants in a Flip Teaching MOOC
}

\author{
Tomás Alcalá Nalváiz , Ángel Fidalgo Blanco², Marta Gómez Gómez³, Inmaculada Gómez Ibáñez ${ }^{4}$, María Luisa \\ Sein-Echaluce Lacleta ${ }^{4}$ \\ jtalcala@unizar.es, angel.fidalgo@upm.es,magomez@usj.es, igomez@unizar.es, mlsein@unizar.es
}

\author{
${ }^{1}$ Depto. Métodos \\ Estadísticos \\ Universidad de Zaragoza \\ Zaragoza, España
}

\author{
${ }^{2}$ Depto. Ingeniería Geológica y \\ Minera \\ Universidad Politécnica de Madrid \\ Madrid, España
}

\author{
${ }^{3}$ Escuela de Arquitectura \\ y Tecnología \\ Universidad San Jorge \\ Zaragoza, España
}

\author{
${ }^{4}$ Depto. Matemática \\ Aplicada \\ Universidad de Zaragoza \\ Zaragoza, España
}

\begin{abstract}
Resumen- Normalmente no se conocen datos precisos sobre la tipología de los participantes en cursos online de formación en abierto (mucho menos si estos son masivos) y de sus motivaciones para seguirlos. Tampoco se dispone de elementos que nos permitan saber si, al finalizar el curso los participantes han interiorizado los elementos característicos que les permite obtener una capacitación de, por ejemplo, un modelo de innovación docente, que le permita implantar experiencias previas que sean sostenibles y transferibles en su labor docente si se trata de profesorado. Por ello, mediante dos encuestas realizadas a los participantes en un MOOC sobre el modelo de Aula Invertida, se estudian los perfiles de los participantes, así como su motivación para realizar el curso y su grado de satisfacción final. La aplicación del método "MAIN: Método de Aplicación de la Innovación educativa" y la información obtenida a través de esas herramientas facilitará el diseño de un curso abierto adaptado a distintas motivaciones y perfiles. De esta forma en las próximas ediciones del curso, este tendrá itinerarios adaptados a los distintos perfiles motivacionales.
\end{abstract}

\section{Palabras clave: Aula invertida, MOOC, Aprendizaje personalizado}

Abstract- Normally, we do not know precise data on the typology of the participants in open online training courses (much less if they are massive) and their motivations to follow them. Nor do we have elements that allow us to know whether, at the end of the course, participants have internalized the characteristic elements that allow them to obtain training in, for example, a model of teaching innovation, which allows them to implement previous experiences that are sustainable and transferable in their teaching work if they are teachers. Therefore, by means of two surveys carried out to the participants in a MOOC on the Inverted Classroom model, the profiles of the participants are studied, as well as their motivation to take the course and their degree of final satisfaction. The application of the method "MAIN: Method of Application of Educational Innovation" and the information obtained through these tools will facilitate the design of an open course adapted to different motivations and profiles. In this way, in future editions of the course, it will have itineraries adapted to different motivational profiles.

\section{Keywords: Flip Teaching, MOOC, Adaptive learning}

\section{INTRODUCCIÓN}

Todos los cursos en abierto, tanto los que incluyen contenidos y actividades para autoaprendizaje (como la iniciativa OCW: OpenCourseWare, (Fidalgo-Blanco, Sein-
Echaluce, \& García-Peñalvo, 2019) como los que además incluyen actividades de apoyo online por parte del profesorado (iniciativa MOOC: Massive Open Online Course, (GarcíaPeñalvo, Fidalgo-Blanco, \& Sein-Echaluce, 2017), presentan una gran heterogeneidad respecto al alumnado (Lerís, SeinEchaluce, Hernández, \& Fidalgo-Blanco, 2016), sin embargo el curso se diseña para un perfil concreto de alumnado (SeinEchaluce, Fidalgo-Blanco, \& García-Peñalvo, 2017)

La primera edición del curso se realiza a ciegas; es decir, no se conoce con exactitud el perfil de los participantes el curso, ni sus objetivos profesionales (respecto a la temática del curso), ni la motivación que les ha llevado a realizarlo.

Por esta razón, los cursos se diseñan para un perfil de público objetivo (por ejemplo, profesorado), para un nivel formativo (por ejemplo, profesorado universitario) y para una motivación y objetivos concretos (por ejemplo, aplicar los contenidos y actividades en su docencia). Así pues, los participantes se deben adaptar al diseño preestablecido del curso,

Si los inscritos tienen el mismo perfil y motivación previstos en el curso, es posible que este les sea útil y de interés, lo que les animará a finalizar el curso. Sin embargo, para los inscritos que no coinciden ni en perfil, ni en motivación el curso no será de interés y por tanto se producirá una alta tasa de abandono. Situación que ocurre habitualmente en los cursos MOOC (Brahimi \& Sarirete, 2015).

Para salvar esta situación existen los cursos MOOC adaptativos, en los que se ofrecen contenidos y actividades acordes con los perfiles y objetivos de los participantes. Sin embargo, el diseño de estos cursos requiere un alto esfuerzo por parte del profesorado creador del curso, ya que debe dominar técnicas y métodos de adaptatividad y contar con tecnologías específicas (Sein-Echaluce, Fidalgo-Blanco, \& García Peñalvo, 2019).

La temática del curso versa sobre el método de innovación educativa denominado Aula Invertida (AI) y que tiene otras denominaciones como Flip Teaching (Dooley \& Makasis, 2020), Flipped Classroom, etc. El método AI está enmarcado dentro de las metodologías activas y su objetivo es que el alumnado aumente su participación activa y colaborativa dentro de una asignatura académica. 
La idea básica del método AI consiste en que el alumnado aprende la lección teórica o realiza una actividad (denominado "Lección en casa") en su lugar de estudio, y de forma previa a la sesión presencial (o síncrona en el caso de clases online), denominada "Deberes en clase". De esta forma, el profesorado puede dedicar más tiempo a realizar actividades participativas con el alumnado, en lugar de transmitir los conceptos teóricos solamente mediante sesiones magistrales.

El método AI ha sido uno de los métodos más utilizados en el último año, de una u otra forma, por la necesidad de realizar formación online debido a la pandemia COVID-19. Aunque, en ocasiones, una interpretación simplista del método AI, como que el alumnado aprende la teoría mediante un video y luego la aplica perfectamente en la sesión síncrona, ha provocado no pocas decepciones en profesorado y alumnado al no aplicarse adecuadamente.

Una forma de comprobar que el método de innovación educativa empleado, en este caso AI, es una buena práctica, se basa en comprobar que es sostenible (que el profesorado la continuará aplicando tras haberla realizado una primera vez) y que es transferible (que se puede utilizar en distintos ámbitos y temáticas educativas). (Fidalgo-Blanco, Sein-Echaluce, \& García-Peñalvo, 2020)

La transferibilidad es una característica asociada a la innovación educativa y que posee el método AI, lo que le hace utilizable en distintos contextos educativos y, por tanto, le permite adaptarse a distintos perfiles de usuario.

En relación a las publicaciones sobre el método AI, en la mayoría de los artículos publicados hasta ahora sobre motivación y satisfacción al utilizarlo (Mendaña-Cuervo, PoyCastro, \& López-González, 2019; Salcines-Talledo, Bemposta, González-Fernández, \& Fuente, 2020) destacan la percepción del alumnado de diferentes titulaciones acerca de esta metodología con respecto a otras utilizadas en su aprendizaje. Se añade que el uso de cuestionarios, incluidos en esta metodología, avalan positivamente sus experiencias en cuanto a las competencias adquiridas.

Por otra parte, la mayoría de las publicaciones sobre cursos MOOC se centran más en discutir su potencial para ofrecer educación de calidad en todo el mundo y a gran escala, que en la medición rigurosa de los resultados de aprendizaje (CastañoGarrido, Maiz-Olazabalaga, \& Garay-Ruiz, 2015). También hay muchos estudios en los que se analizan los perfiles de usuarios de los MOOC, coincidiendo en que hay una gran variedad de países de origen, profesiones, edades, estudios previos y objetivos. Y también se estudia la alta tasa de abandono, como característica de ese tipo de cursos. (Garg \& Goel, 2021)

En este trabajo se describirá además del perfil de las personas que participaron en un MOOC sobre AI, la posibilidad de que el curso sea útil para distintos perfiles y distintas motivaciones. Lo que permitirá ampliar el espectro de posibles usuarios.

En cuanto a la posibilidad de que un curso de formación abierta pueda satisfacer distintas motivaciones, se aplicará el método MAIN: Método de Aplicación de la Innovación educativa (Fidalgo-Blanco \& Sein-Echaluce, 2018). En este método se describe una visión funcional del método de Aula Invertida que puede servir para conocer qué es, plantear la posibilidad de aplicarlo y aplicarlo de forma inmediata.
Partiendo de un curso en abierto dirigido a alumnado hispanohablante, se han realizado dos encuestas sobre motivación y satisfacción. Los resultados que de este estudio se obtengan pueden orientar a otros formadores en el diseño y estructuración de nuevos cursos en abierto. Además, el conocimiento sobre los perfiles permite generar itinerarios adaptados a las necesidades del alumno.

Así pues, las hipótesis /objetivos que se plantean en este trabajo son las siguientes:

- Es posible realizar un diseño instruccional relativo a varios perfiles de usuario y motivaciones dentro de los cursos MOOC.

- Es posible aplicar de forma práctica el método AI, cumpliendo las características de buena práctica de innovación educativa.

Este trabajo permite diseñar un curso con el modelo propuesto y que, con la información obtenida pueda adaptarse a distintos perfiles, que responda a varias motivaciones (desde un interés general hasta la aplicación inmediata), y que permita, además de aplicar el método, realizar una buena práctica.

Los siguientes apartados incluyen la descripción del modelo que se propone aplicar para diseñar el curso abierto personalizado. A continuación, se describen las herramientas utilizadas para el estudio de los perfiles, motivación y satisfacción de los participantes en un MOOC sobre AI, seguido de los resultados y las conclusiones.

\section{MODELO DE CURSO PROPUESTO}

El método MAIN permite estructurar un curso de formación que aplique cualquier método de innovación educativa y que pueda ser utilizable por distintos perfiles de usuarios, ámbitos, áreas de conocimiento y objetivos (Sein-Echaluce, FidalgoBlanco, \& García-Peñalvo, 2019; Sein-Echaluce, FidalgoBlanco, García-Peñalvo, \& Balbín, 2020). Este método contempla un conjunto de fases para conseguir mejoras de aprendizaje en el aula. Concretamente, la fase 3 de dicho método indica cómo estructurar la enseñanza de cualquier método de innovación educativa para que se pueda aplicar en distintos ámbitos académicos y áreas de conocimiento y que pueda satisfacer diversos objetivos académicos, desde conocer hasta aplicar.

La fase 3 de MAIN permite describir un flujo de procesos (procesos, relación y secuencia) que intervienen en el método AI (en nuestro caso). La Figura 1 muestra el flujo de procesos para las actividades previas a la sesión presencial (es decir, durante la "Lección en casa") y que seguirá el alumnado para realizar el método (1A hasta $3 \mathrm{~A})$, la secuencia de actividades conjunta entre profesorado y alumnado (1PA) y la secuencia de procesos que únicamente realizará el profesorado (1P y $2 \mathrm{P})$.

De esta forma se puede conocer tanto el funcionamiento del método AI como la forma de aplicarlo. Para aplicar el método AI se describe el contenido, tecnología y actividades que conlleva cada proceso. 


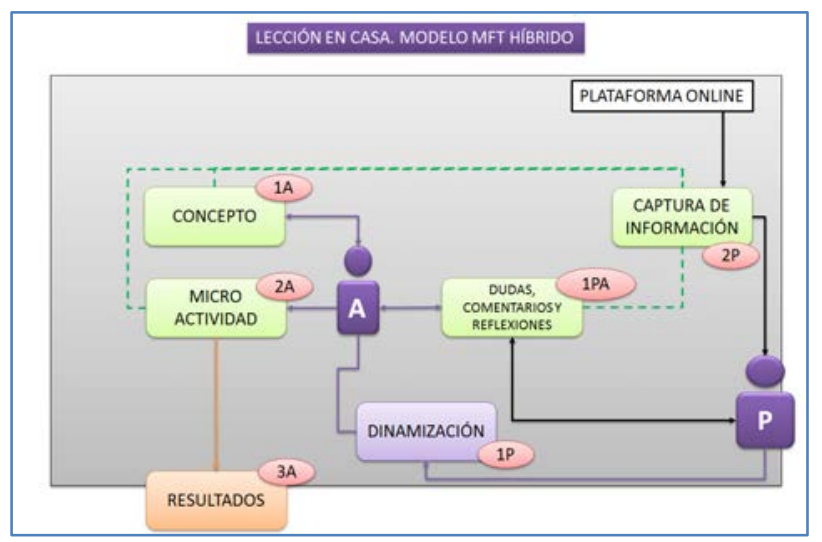

Figura 1. Ejemplo de flujo de procesos para las actividades previas en el método AI.

En la tercera fase del método MAIN para cualquier innovación educativa, se estructuran los contenidos tenidneo en cuenta estos aspectos:

- Estrategia; actividades a realizar por el profesorado y alumnado

- Tecnología: funcionalidad y características que debe tener la tecnología a utilizar.

- Contenidos: tipología y formato del contenido.

El método de innovación educativa, en este caso el método AI, se divide en tres módulos:

- Los contenidos. Se refiere a la tipología del contenido que aplica el método AI. Un ejemplo de tipología es el "video". La tipología de contenidos no enseña cómo desarrollar contenidos en ese formato, pero permite que el profesorado conozca el esfuerzo a realizar para transformar (o desarrollar) sus contenidos en dicha tipología. Aunque el método MAIN no incluye formación sobre cómo desarrollar la tipología de contenidos, se suelen incluir guías para realizarlo e información ampliada con formación específica para conseguirlo. En el caso del método AI, se trabaja con diversas tipologías de contenidos, la mayoría de ellas muy conocidas por el profesorado (apuntes, presentaciones, capítulos de libros, noticias, casos, etc.). No debe olvidarse la calidad de los contenidos en cualquier modalidad de enseñanza y, en particular, en la formación virtual.

- La tecnología. Aunque el curso se puede centrar en una tecnología concreta, lo que se describe es la funcionalidad de la tecnología. Se describen, por tanto, las características que debe tener la tecnología para ser usada en el método AI. De esta forma, el usuario podrá utilizar una tecnología u otra, por ejemplo, la que le sea más familiar, la que suministre el centro formativo, la más barata o la que sea más popular.

- La Estrategia. Son las actividades que tiene que realizar tanto el profesorado como el alumnado.

\section{DESCRIPCIÓN DE LAS HERRAMIENTAS}

En esta sección se incluyen las herramientas que permitirán obtener los resultados del estudio, el material y métodos, la encuesta de motivación y la encuesta de satisfacción y valoración del modelo.

\section{A. Material y métodos}

En el curso MOOC : 'Flip Teaching: una metodología activa' implementado en la plataforma MiriadaX, se habilitaron dos encuestas voluntarias, una inicial sobre motivación y otra final sobre satisfacción que fueron cumplimentadas entre marzo de 2018 y junio de 2019. Debido al carácter anónimo de estos recursos, se solicitaba un código en ambas encuestas que permitiera su posterior emparejamiento.

Se recogen una serie de datos demográficos generales, tales como País (abierta), Género (masculino/femenino) y Edad. Respecto al perfil formativo se preguntaba el nivel de estudios finalizados (5 categorías: Educ. Primaria, Educ. Secundaria, Formación Profesional, Educ. Superior: Grado/Licenc, Educ. Superior: Master/Doctorado) y respecto al Perfil profesional (9 categorías: Autónomo, Empleado No Docente, Estud. No universitario, Estud. Univ. (No área educación), Estud. Univ. (Área Educ.), Docente de Ed. Infantil/Primaria (hasta 12 años), Docente Formación Profesional, Docente Secund./Bachiller., Docente Universitario)

\section{B. Encuesta de Motivación}

En la encuesta inicial se incluyen siete cuestiones sobre la motivación del alumno a la hora de hacer este curso. Configuran un instrumento propio y específico en el ámbito del método Flip Teaching (FT o AI en español). Son redactadas en base al interés de los profesores responsables del curso y de su experiencia previa en cursos sobre AI. Cada pregunta admite tres respuestas: $\mathrm{Si} / \mathrm{No} / \mathrm{No}-$ contesta.

Las preguntas sobre motivación son:

- (M1) Conocer qué es el modelo AI, aunque de momento no voy a aplicarlo

- (M2) Comenzar a aplicar el modelo AI.

- (M3) Tener una nueva visión del modelo AI

- (M4) Ver cómo está organizado el curso y/o acceder a los materiales del mismo

- (M5) Por interés profesional/laboral

- (M6) Ampliar mi formación en términos generales

- (M7) Obtener el certificado de superación del curso

\section{Encuesta de satisfacción y valoración del modelo}

Esta encuesta se realiza tras haber finalizado el curso MOOC y sigue siendo de carácter voluntario. Tiene una primera parte sobre el grado de cumplimiento de los 6 módulos en los que se divide el curso (Mod 0: Presentación, Mod 1: Qué es y qué no es Flip Teaching, Mod 2: Trabajando con el video, Módulo 3: Micro Flip Teaching: un método activo. El Modelo, Mod 4: Aplicación: Tecnologías, estrategias y ejemplos, Mod 5: Impacto. Publicación científica y medición de resultados). Se pregunta si se ha completado cada módulo, mediante cuestión cerrada con dos opciones Completado/No-completado.

La segunda parte consta de 8 preguntas referentes al grado de acuerdo con una serie de afirmaciones relativas al modelo AI que se propone. Cada pregunta está formulada en forma de escala Likert de 4 puntos, ordenados desde "nada de acuerdo" hasta "totalmente de acuerdo", se admite también la opción "No sabe/No contesta". Finalmente, se incluyó una pregunta sobre el grado alcanzado respecto a las expectativas iniciales, 
puntuada de 1 (nada) a 5 (totalmente). Preguntas de acuerdo con el modelo propuesto:

(V1) Los componentes funcionales se pueden utilizar como una guía para aplicar una experiencia AI en diferentes contextos.

(V2) El enfoque de aplicación de las tecnologías en base a la misión del componente funcional permite conocer de antemano el efecto que va producir su aplicación.

(V3) El conjunto formado por los componentes funcionales, la tecnología y la estrategia, es una herramienta útil para realizar experiencias $A I$

(V4) Sin variar los componentes funcionales y la tecnología, distintas estrategias permiten diferentes enfoques de planificación de una experiencia AI.

(V5) Focalizar la atención en el objetivo de la tecnología facilita su sustitución por futuras tecnologías.

(V6) La identificación de los componentes funcionales y su interrelación facilita la innovación metodológica del modelo AI.

(V7) Identificar los componentes funcionales, la tecnología y la estrategia de una experiencia AI permite su transferencia entre distintas asignaturas.

(V8) Las consideraciones realizadas en este curso sobre la forma de realizar una experiencia AI para su divulgación cientifica facilita su publicación.

Se realizó un proceso de depuración de encuestas, eliminando duplicados (personas que responden dos o más veces) e inconsistencias. Se analizaron descriptivamente los datos relativos al perfil demográfico, de formación y ocupación.

El perfil motivacional se analizó mediante la creación de grupos homogéneos basado en un análisis bicluster sobre las 8 preguntas de motivación. Se permitió un máximo de 15 grupos y criterio de selección automático basado en criterio de información bayesiano (BIC). Esta metodología genera grupos homogéneos e identifica las variables más relevantes en su agrupación. Tras un primer análisis, se detectaron grupos totalmente homogéneos con respuestas SI o NS/NC a todas las motivaciones. Fueron eliminados de la segunda etapa que se resume aquí. Se analizó la relación entre los grupos homogéneos y las variables demográficas, de formación y de ocupación.

En la encuesta final se analizaron los porcentajes de cumplimentación de cada uno de los 6 módulos en los que está dividido el curso. Para los ítems de valoración del modelo se analizó la consistencia interna mediante el alpha de Cronbach. Se midió el grado de correlación de la puntuación agregada con en el grado de expectativas alcanzado.

Con los ítems de valoración del modelo AI también se repitió un análisis clúster bietápico que permitiera establecer diferentes perfiles de satisfacción. Se consideraron sólo encuestas completas en estos 8 ítems. Todos los cálculos estadísticos se realizaron con SPSS 25.0.

\section{Resultados}

El número de encuestas iniciales válidas tras el proceso de depuración fue de 1867. Los participantes que respondieron fueron mayoritariamente mujeres $(64,8 \%)$. Por nacionalidades, la mayor frecuencia es española (53\%) seguidos de Ecuador, México, Perú, Colombia y Argentina (todos ellos acumulan el $85,5 \%$ de respuestas). En cuanto a estudios, es mayoritaria la Formación universitaria de grado $(46.5 \%)$ que aumenta al $79,8 \%$ si se amplía con máster o doctorado. El perfil profesional dominante es el profesorado de educación secundaria $\mathrm{o}$ bachillerato $(27,7 \%)$ seguido del docente universitario $(19,2 \%)$ $\mathrm{y}$ en general los docentes representan el $75,4 \%$ de las respuestas. La edad media es 41 años ( $\mathrm{DT}=11,3$ años).

En un primer análisis de motivación, se detectan dos grupos de individuos con todas sus respuestas homogéneas en todas las preguntas (SI en todo o NS/NC en todos los ítems). Debemos entenderlos como personas que no desean declarar su motivación. Se procede a un segundo análisis de grupos con las 1124 encuestas restantes. El algoritmo identifica 5 perfiles (ver Figura 2) de motivación diferentes, que resumimos con sus respectivas frecuencias

P1(21,1\%) motivados por AI con cierta urgencia en su aplicación inmediata

P2(19,8\%) interesados por aplicar AI pero no en busca de materiales

P3(22,6\%) interesados de forma general por todo lo que ofrece el curso, incluida su certificación, pero sin interés inmediato en aplicar AI.

P4(24,8\%) atraídos por el curso, sin interés inmediato en aplicar AI y sobre todo no interesados por la certificación del curso

P5(11,7\%) atraídos por la formación MOOC en general, por interés profesional, pero no especialmente por una nueva visión de AI.

En el cruce de las agrupaciones con las variables demográficas y de ocupación destacamos que el perfil P1 tiene mayor diversidad de nacionalidades sin tanto dominio de la española $(21,5 \%)$, formación de grado $(50,6 \%)$ y dedicación profesional como docente de secundaria/bachiller $(37,1 \%)$.

En la encuesta final se validan 1050 cuestionarios. El perfil por nacionalidades y por ocupación se mantienen homogéneos ( $\mathrm{p}$-valor del contraste chi-cuadrado $>0,05$ ). La edad media de las personas que responde también es homogénea ( $p$-valor $>$ 0,05 del t-test). Sin embargo, hay una disminución relativa de mujeres que no responden a la encuesta final y de personas con estudios de formación profesional que tampoco responden la encuesta final ( $p$-valor $<0,05$ en las correspondientes pruebas chi-cuadrado).

Respecto al análisis de módulos completados, el 91,3\% declaran haber completado todos los módulos (no se implementó un mecanismo de control específico para esta cuestión). Entre los que no completaron todos los módulos (ver Figura 3), destaca el no haber terminado el módulo 5, orientado al Impacto y publicación de resultados sobre AI (sólo completado por un $23 \%$ ) seguido del módulo orientado al uso del vídeo en AI (ya con un $80 \%$ de personas de este grupo que lo completan).

Para el análisis de la escala piloto sobre la valoración del modelo AI propuesto, se consideraron únicamente aquellas personas que contestaron a los 8 ítems del cuestionario y que forman una muestra de 661 respuestas. La consistencia interna medida por el alpha de Cronbach da un valor de 0,867 , que se 
puede considerar como alta o muy alta. Agregando las puntuaciones de los 8 ítems, se obtiene un valor mínimo de 8 y máximo de 32 , con una media de $27,4(\mathrm{DT}=3,66)$. Un análisis de grupos sobre estas respuestas revela un subgrupo minoritario $(1.8 \%)$ con opiniones en desacuerdo en todos los ítems y otro subgrupo más numeroso $(30.4 \%)$ con un acuerdo absoluto o casi absoluto en todos los ítems, además de otros tres subgrupos con puntuación media intermedia y diferenciados por los ítems V3 y V6 de esta escala; sobre la innovación metodológica del modelo y la utilidad del modelo para realizar experiencias.

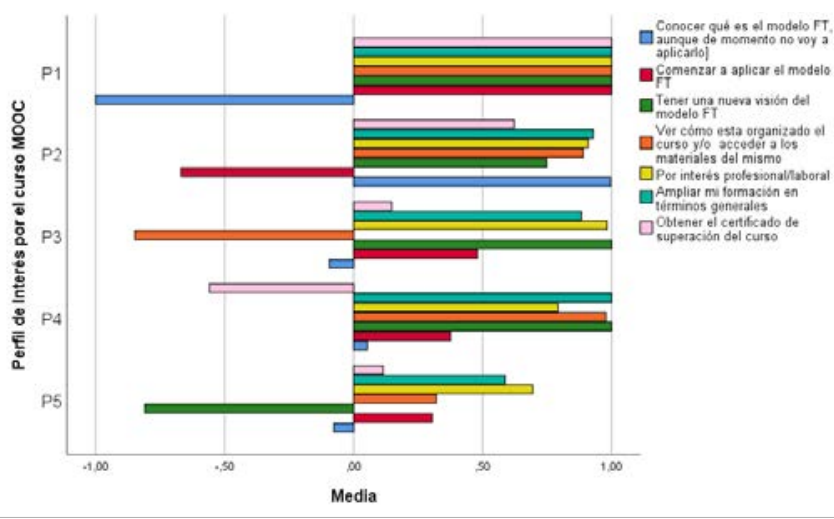

Figura 2. Gráfico de barras para cada uno de los perfiles identificados. Valores medios (se ha utilizado la codificación $\mathrm{NO}=-1, \mathrm{SI}=1$, $\mathrm{NS} / \mathrm{NC}=0$ )

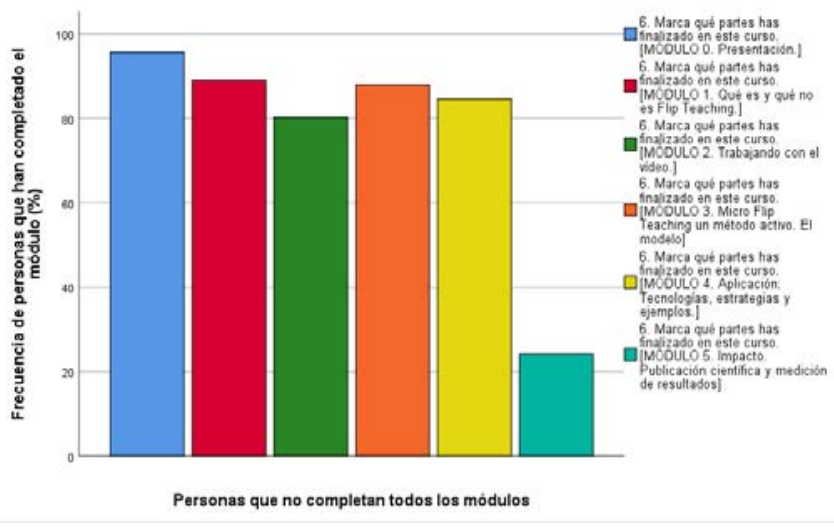

Figura 3. Entre los que no completan todos los módulos, distribución de porcentajes de módulos sí completados.

La variable sobre el cumplimiento de expectativas toma un valor de 4,24 (sobre 5) con DT=0,80. La correlación entre la puntuación total de la escala y la expresada directamente sobre el grado de expectativas alcanzado en el curso es de 0,4; positiva pero moderada. En la Figura 4 se puede apreciar la relación entre los intervalos de confianza (IC al 95\%) para el valor medio de la escala de acuerdo con el modelo AI para cada uno de los niveles de expectativas alcanzadas. Los que más acuerdo con el modelo AI reconocen, también son los más satisfechos en sus expectativas están tras el curso.

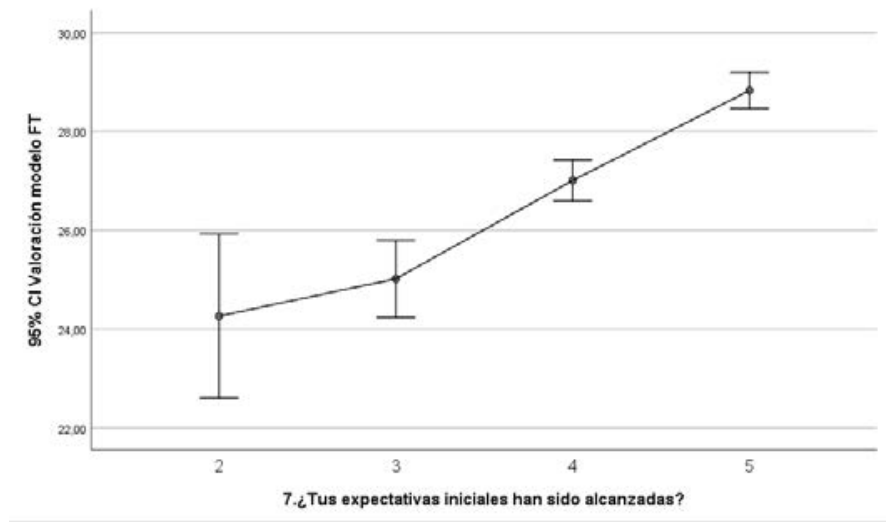

Figura 4. Gráfico de barras de error para la valoración agregada del modelo AI (FT) en función de las expectativas iniciales alcanzadas.

\section{CONCLUSIONES}

A través de los dos cuestionarios autocompletados por los participantes hemos constatado una fuerte necesidad de estos cursos en abierto sobre metodología AI en el espacio hispanohablante. El mayor interés ha sido mostrado por docentes de secundaria/bachiller y universidad. Entre sus motivaciones destaca la necesidad de formarse en AI para poner en práctica esta metodología, incluso cuando no se busca un reconocimiento académico. Aproximadamente el $12 \%$ se acerca a este tipo de cursos con una motivación de formación general, especialmente si se acompaña de un reconocimiento o acreditación. La detección de estos perfiles permitirá rediseñar este curso o similares y diversificar los contenidos y exigencias en función de los intereses de los potenciales alumnos.

El alumno suele completar un alto porcentaje de los módulos, y hemos identificado el módulo sobre impacto y publicación que no resulta de tanto interés y, por tanto, susceptible de ser un módulo optativo o sustituido por otro de mayor interés (como ejemplos concretos de aplicación del método, lecciones aprendidas de dichos ejemplos, etc.)

El cuestionario de satisfacción o acuerdo con la metodología AI se puede utilizar como una escala de alta consistencia interna, donde un porcentaje del $2 \%$ se queda en la parte baja de la escala y en torno a un $30 \%$ de las contestaciones se sitúan en la parte alta de la escala.

Por otro lado, tras el seguimiento y visionado de los módulos se consigue que los alumnos del curso comprendan el potencial de identificar las componentes funcionales, la tecnología y la estrategia a la hora de poder implementar experiencias de AI que sean sostenibles y transferibles entre asignaturas. Además, cuando el modelo de AI propuesto se identifica correctamente, la tecnología puede ser variada sin alterar la utilidad de la experiencia de AI. El punto sobre difusión y publicación de resultados de experiencias de AI es el que se ve con mayor dificultad y será necesario insistir o añadir recomendaciones en este sentido. Las expectativas iniciales del alumnado son satisfechas con una alta valoración.

Estos resultados muestran la diversidad de motivaciones de los participantes en un curso abierto sobre AI. Así mismo, se muestra que dichas motivaciones son satisfechas de una forma general, en el MOOC sobre AI. por lo que los participantes cuya motivación era aplicar el método AI, han quedado satisfechos (luego se cumple el objetivo 2). Así mismo, ambas 
circunstancias avalan la necesidad de realizar cursos abiertos que se adapten a distintos perfiles y motivaciones, para así aumentar el grado de satisfacción de los participantes y disminuir la tasa de abandono de este tipo de cursos. Los resultados de este trabajo ya dan indicaciones sobre los tipos de perfil /motivación que habría que tener en cuenta al diseñar un curso con esas características (objetivo 1).

Tras detectar los perfiles más frecuentes de los usuarios en los cursos sobre AI y con el modelo MAIN descrito, el siguiente paso es rediseñar y adaptar los contenidos del curso en abierto en función de las necesidades y requerimientos de los potenciales participantes. Próximamente se publicará un curso OCW (OpenCourseWare) de la Universidad de Zaragoza (ocw.unizar.es) donde los autores han aplicado parcialmente los resultados de este trabajo.

\section{AgRAdECIMIENTOS}

Este trabajo ha sido financiado por el Vicerrectorado de Política Académica de la Universidad de Zaragoza a través del proyecto PRAUZ 19_326 y por el Servicio de Innovación Educativa UPM-Convocatoria MOOC Flip Teaching: Una metodología activa.

\section{REFERENCIAS}

Brahimi, T., \& Sarirete, A. (2015). Learning outside the classroom through MOOCs. Computers in Human Behavior, 51(PB), 604-609. https://doi.org/10.1016/j.chb.2015.03.013

Castaño-Garrido, C., Maiz-Olazabalaga, I., \& Garay-Ruiz, U. (2015). Diseño, motivación y rendimiento en un curso MOOC cooperativo. Grupo Comunicar, 22(44), 19-26. https://doi.org/10.3916/C44-2015-02

Dooley, L., \& Makasis, N. (2020). Understanding Student Behavior in a Flipped Classroom: Interpreting Learning Analytics Data in the Veterinary Pre-Clinical Sciences. Education $\quad$ Sciences, 260. https://doi.org/10.3390/educsci10100260

Fidalgo-Blanco, Á., Sein-Echaluce, M. L., \& García-Peñalvo, F. J. (2020). Hybrid Flipped Classroom: Adaptation to the COVID situation. ACM International Conference Proceeding Series. https://doi.org/10.1145/3434780.3436691

Fidalgo-Blanco, Ángel, Sein-Echaluce, L. M. L., \& GarcíaPeñalvo, F. J. (2019). D-OCW. Un nuevo modelo para desarrollar cursos $O C W$ dinámicos, sociales y adaptados a necesidades reales. https://doi.org/10.5281/ZENODO.3436491

Fidalgo-Blanco, Ángel, \& Sein-Echaluce, M. L. (2018). Método MAIN para planificar, aplicar y divulgar la innovación educativa. Education in the Knowledge Society (EKS), 19(2), 83-101. https://doi.org/10.14201/eks201819283101

García-Peñalvo, F. J., Fidalgo-Blanco, Á., \& Sein-Echaluce, M. L. (2017). Los MOOC: Un análisis desde una perspectiva de la innovación institucional universitaria.
La Cuestión Universitaria, O(9), 117-135. Retrieved from

http://polired.upm.es/index.php/lacuestionuniversitaria/a $\mathrm{rticle} / \mathrm{view} / 3583 / 0$

Garg, M., \& Goel, A. (2021). A Data-Driven Approach for Peer Recommendation to Reduce Dropouts in MOOC. Lecture Notes in Electrical Engineering, 735 LNEE, 217-229. https://doi.org/10.1007/978-981-33-6977-1_18

Lerís, D., Sein-Echaluce, M. L., Hernández, M., \& FidalgoBlanco, A. (2016). Heterogeneous users in MOOC and their adaptive learning needs. Education in the Knowledge Society, 17(4). https://doi.org/10.14201/eks201617491109

Mendaña-Cuervo, C., Poy-Castro, R., \& López-González, E. (2019). Metodología Flipped Classroom: Percepción de los alumnos de diferentes Grados universitarios. Innoeduca. International Journal of Technology and Educational Innovation, 5(2), 178-188. https://doi.org/10.24310/INNOEDUCA.2019.V5I2.5223

Salcines-Talledo, I., Bemposta, E. C., González-Fernández, N., \& Fuente, J. R. V. (2020). Estudio de caso sobre las percepciones de los estudiantes respecto al modelo Flipped Classroom en asignaturas de ingeniería. Diseño e implementación de un cuestionario. Revista Complutense de Educación, 31(1), 25-34. https://doi.org/10.5209/RCED.61739

Sein-Echaluce, M., Fidalgo-Blanco, Á., \& García-Peñalvo, F. J. (2019). Diseño de un proyecto de innovación educativa docente a partir de indicadores transferibles entre distintos contextos. In Aprendizaje, Innovación y Cooperación como impulsores del cambio metodológico. Actas del V Congreso Internacional sobre Aprendizaje, Innovación y Competitividad. CINAIC 2019 (9-11 de Octubre de 2019, Zaragoza, España) (1st ed.). Zaragoza: Servicio de Publicaciones Universidad de Zaragoza. https://doi.org/10.26754/cinaic.2019

Sein-Echaluce, Maria Luisa, Fidalgo-Blanco, A., \& García Peñalvo, F. J. (2019). Innovative trends in flipped teaching and adaptive learning. Preface (Maria Luisa Sein-Echaluce, A. Fidalgo-Blanco, \& F. J. García Peñalvo, Eds.). Hershey, PA, USA: IGI GLOBAL. https://doi.org/10.4018/978-1-5225-8142-0

Sein-Echaluce, María Luisa, Fidalgo-Blanco, Á., \& GarcíaPeñalvo, F. J. (2017). Adaptive and cooperative model of knowledge management in MOOCs. Lecture Notes in Computer Science (Including Subseries Lecture Notes in Artificial Intelligence and Lecture Notes in Bioinformatics), $10295 \quad$ LNCS, 273-284. https://doi.org/10.1007/978-3-319-58509-3_22

Sein-Echaluce, María Luisa, Fidalgo-Blanco, Á., GarcíaPeñalvo, F. J., \& Balbín, A. M. (2020). Global Impact of Local Educational Innovation. Lecture Notes in Computer Science), 12205 LNCS, 530-546. Springer. https://doi.org/10.1007/978-3-030-50513-4_39 